\title{
Ciencias sociales y salud en México: movimientos fundacionales y desarrollos actuales
}

\author{
Social sciences and health in M exico: \\ founding schools and current trends
}

Roberto Castro 1

\footnotetext{
1 Programa de

Investigación Sociedad,

Salud y Centro Regional de Investigaciones Multidisciplinarias (UNAM) Av. Universidad $s / n$ circuito 2, Col. Chamilpa, c.p. 62210, Cuernavaca, M or. rcastro@servidor.unam.mx
}

Abstract This paper describes the main developments of social sciences applied to health in M exico. It begins with a brief description of the three founding schools that have taken health as their subject matter: medical anthropology, with a long tradition in this country; social medicine, developed basically by the $M$ etropolitan Autonomous U niversity at Xochimilco; and the sociology of public health, promoted mainly by the N ational Institute of Pub$\mathrm{lic} \mathrm{H}$ ealth. After reviewing the main contributions of these schools of thought, and the main debates that have taken place among them, this paper focuses on the main contemporary developments, a sort of "second generation" of M exican medical sociology. This review distinguishes between theoretical, methodological, and substantive contributions, the last ones being those related to the most dynamic and productive fields. The paper concludes by pointing out the relevance of medical sociology in $M$ exico and by arguing on the need to reactivate the academic exchange and debate among the different schools, in order to increase the quality of the scientific production in this field. Key words M edical sociology, M edical anthropology, Public health, Reproductive health, $\mathrm{H}$ ealth policies and health services utilization
Resumen En este trabajo se describen algunos de los principales desarrollos de las ciencias sociales aplicadas a la salud en M éxico en los últimos 25 años. El artículo inicia con una breve descripción de las tres corrientes fundacionales que han hecho dela salud su objeto de estudio: la antropología médica, de larga tradición en este país; la medicina social, impulsada principalmente desde la U niversidad Autónoma M etropolitana-Xochimilco; y la sociología de la salud pública, impulsada desde el Instituto N acional de Salud Pública. Tras revisar los principales aportes de cada corriente, así como los debates que entre ellas ha tenido lugar; describe los desarrollos recientes en este campo, mismos que constituyen una suerte de "segunda generación" de la sociología médica mexicana. La revisión se centra en los aportes en el campo de lo teórico, lo conceptual, y las áreas con más producción actual. Concluye señalando la importancia de este campo en M éxico y la necesidad de reactivar los intercambi os académicos entre las diferentes corrientes de pensamiento, como mecanismo para elevar la calidad de los trabajos.

Palabras-claves Sociología médica, Antropología médica, Salud pública, Salud reproductiva, Políticas y utilización de servicios de salud 


\section{Introducción}

La reflexión sobre los problemas de salud desde una perspectiva social constituye una práctica académica que ha existido por varias décadas en M éxico. Diversos estudios han acreditado la existencia de una tradición tanto sociológica (Laurell, 1974; Almada, 1986) como antropológica (Aguirre-Beltrán, 1986; M enéndez, 1990) en el estudio de los problemas de la salud, que se manifiesta ya con mucha fuerza por lo menos desde la década de los sesentas, si bien en sus comienzos de manera irregular y heterogé nea. Es posible identificar varias "tradiciones" 0 escuelas en el origen del pensamiento social en salud en M éxico. Estas escuelas mantienen entre sí diferencias importantes no sólo por los marcos teóricos que eligen para conceptualizar los problemas que estudian, sino también por las posiciones políticas que adoptan en su quehacer científico. Esas diferencias las han Ilevado a sostener intensos debates sobre el origen de las desigual dades en salud de este país, así como sobre las políticas públicas que se implementan para resolverlas.

En este trabajo se describen algunos de los principales desarrollos de las ciencias sociales aplicadas a la salud en M éxico, particularmente durante los últimos 25 años. El trabajo no pretende ser exhaustivo. Por el contrario, el énfasis está puesto únicamente en los principales autores y en los aportes más destacables. Ello implica reconocer de entrada la injusticia que por omisión o simplificación se tendrá que hacer a los numerosos investigadores que actualmente realizan, directa o indirectamente, investigación social en salud. En la primera parte se caracteriza brevemente a las principales corrientes fundacionales del pensamiento social en salud en M éxico y se enfatizan los principales aportes teóricos y metodológicos de cada escuela, así como los principales debates que sostuvieron entre ellas. En la segunda parte del trabajo se hace una caracterización de la situación actual de la sociología médica en M éxico, enfatizando los principales temas que concentran la atención del grueso de los investigadores en este campo y señalando, en lo posible, algunos de los principales aportes que las ciencias sociales han hecho, en este país, al estudio de los problemas de la salud y la enfermedad de la población.

Para entender mejor lo que sigue, es importante tomar en cuenta uno de los rasgos distintivos de las ciencias sociales en salud en la actualidad en M éxico: su carácter eminentemen- te latinoamericano. Este atributo se debe no sólo a que M éxico comparte la misma lengua (el español) con casi todos los países de América Latina (excepto Brasil), y una historia muy semejante en tanto ex-colonias de España y Portugal; se debe también al hecho de que durante la represión militar que tuvo lugar en muchos países del Cono Sur durante las décadas de los setentas y los ochentas, muchos investigadores de esos países emigraron a M éxico buscando preservar su vida académica. Las ciencias sociales aplicadas a la salud se vieron particularmente enriquecidas con esta inmigración. El carácter latinoamericano de la sociología médica en M éxico explica a su vez que muchos de sus autores prefieran publicar en libros y revistas científicas de M éxico, Brasil, Argentina o Chile, más que en revistas norteamericanas o europeas. A ello se debe, quizás, el relativo desconocimiento que en estos últimos ámbitos prevalece sobre el quehacer científico-social en salud de países como M éxico.

\section{Los origenes}

El pensamiento social en salud en M éxico ha constituido, desde hace muchos años, un campo muy dinámico y creciente, en el que es posible, no sin riesgos de esquematizaciones, distinguir tres escuelas o grupos fundacionales: a) los antropólogos médicos; b) los exponentes de la medicina social, y c) los sociólogos de la salud pública.

\section{La tradición médico-antropológica}

Un primer núcleo de producción teórica y empírica de gran importancia es atribuible al grupo de antropólogos médicos que, con mucho, son herederos de la más antigua tradición de conocimiento en este campo. Sus precursores vivieron en los primeros años de la conquista, como Fray Bernardino de Sahagún (Sahagún, 1989; León-Portilla, 1999), Diego Durán y muchos otros; sus esfuerzos por dilucidar la lógica y las características de la medicina prehispánica se han mantenido vivos a lo largo de los siglos transcurridos desde entonces (Vargas, 1989), principalmente por el grupo de especialistas denominados etnohistoriadores o historiadores médicos, entre los que destaca, entre muchos otros, la obra de Alfredo López-Austin $(1971,1980)$, autor de una de las más importantes reconstrucciones del pensamiento médi- 
co Náhuatl que se hayan escrito hasta la fecha y que ilumina muchas de las prácticas que aún existen en los sectores populares de M éxico.

Los aportes de la antropología médica mexicana a lo largo del siglo 20 han sido objeto de estudios que hoy son ya un referente indispensable. Autores como Aguirre-Beltrán (1963) dieron un impulso decisivo al proceso de re-legitimización de la perspectiva antropológica en el campo de las investigaciones en salud, frente a un panorama de creciente medicalización que centraba en la perspectiva biomédica todo intento de análisis y explicación de los problemas de salud. En las últimas décadas, cabe resaltar el trabajo de varios autores que han orientado el trabajo de las generaciones contemporáneas en este campo. Por ejemplo, Luis Alberto Vargas (Vargas, 1973, 1978, 1991, 1993; Simoni et al., 1983; Vargas et al., 1993; García de Alba et al., 1998) cuyos trabajos han buscado siempre tender puentes no sólo entre la medicina y la antropología, sino también entre los diversos tipos de medicina (moderna, tradicional, etc.) actualmente existentes en el país. Lozoya y Zolla (1983), por su parte, impulsaron una serie de trabajos que buscaban hacer visible lo que la medicina moderna insistía en ignorar: el papel de las prácticas médicas tradicionales entre amplios sectores de la población mexicana (Zolla et al., 1988; M ellado et al., 1989).

$Y$ finalmente, también con carácter fundacional, hay que destacar los trabajos de Eduardo $M$ enéndez que, desdeel Centro de Investigaciones y Estudios Superiores en Antropología Social, ha impulsado desde principios de la dé cada de los ochentas el desarrollo de la antropología médica desde una perspectiva crítica. M enéndez (1978), propuso el concepto de "modelo médico hegemónico" para caracterizar a ese modelo de medicina que devino dominante desde principios del siglo 19, bajo el capitalismo, y que, además de fundamentar jurídicamente su apropiación exclusiva de la enfermedad, privilegió una mirada sobre ella biologista, individualista, ahistórica, asocial, mercantilista y pragmática. El desarrollo del concepto impulsó una serie de estudios sobre las formas en que se articulan las diversas instituciones que actúan sobre la salud y la enfermedad, bien desde una posición de subalternidad ( $M$ enéndez, 1984), o bien desde la situación de "crisis" que vive el modelo médico hegemónico ( $M$ enéndez, 1985a). De igual forma, bajo esta óptica surgie ron diversos estudios sobre al coholización ( $\mathrm{Me}$ néndez, 1985b), sobre auto-atención en salud
(M enéndez, 1983), y sobre la forma en que coexisten las prácticas médicas alternativas (tradicionales y domésticas) junto con la medicina dominante (M ódena, 1990). La antropología médica en M éxico es hoy una corriente vigorosa a la que se deben muchos de las mejores investigaciones sociales en salud (Campos, 1992). Sin embargo, los aportes actual es de esta escuela son objeto de otro artículo en este número, por lo que nos centraremos en las contribuciones de la sociología médica en sus diversas vertientes (medicina social, sociología de la salud pública, y otros enfoques recientes).

\section{La medicina social}

Una segunda corriente fundacional, menos centrada en la antropología y más en la perspectiva sociológica, es la que se desarrolló, desde mediados de la década de los setentas, la M aestría en M edicina Social, de la Universidad Autónoma M etropolitana (U nidad Xochimilco). Junto con investigadores de otros países de América Latina, desde esta institución se ha impulsado la llamada corriente de la medicina social (Laurell, 1989). Esta corriente constituye uno de los mejores ejemplos, a nivel internacional, de los aportes que puede hacer el análisis marxista en cuestiones de salud. En un trabajo bien conocido en la región, Laurell (1975) estableció que el objetivo de la medicina social latinoamericana no es tanto señalar, como lo venían haciendo autores como N avarro (1976) en Estados U nidos, que el sistema capital ista determina la natural eza de las enfermedades de las poblaciones, sino más bien demostrar la manera específica en que dicha determinación tiene lugar. La autora sostiene que la enfermedad no puede ser considerada simplemente como un estado biológico, ni como la consecuencia mecánica de la pobreza. La salud y la enfermedad, sostiene, no pueden ser tomadas como dos entidades separadas, sino que deben ser entendidas como dos momentos (unidos dialécticamente) de un mismo fenómeno. De aquí deriva uno de los principales conceptos de la corriente de la medicina social latinoamericana: más que hablar de salud y de enfermedad separadamente, esta escuela tiene al proceso de saludenfermedad como su objeto de estudio (Laurell, 1982). Años más tarde, M enéndez (1983) sostuvo que también las conductas de los individuos frente a la enfermedad son parte integral del concepto salud-enfermedad, por lo que propone que el concepto debe ser proceso salud-en- 
fermedad-atención. Desde esta perspectiva teórica se desarrolló también un trabajo de análisis de las "necesidades esenciales en salud" en M éxico, que pronto se convirtió en una referencia obligada tanto para investigadores como para estudiantes del tema (Boltvinik et al., 1983).

Dado su enfoque crítico, la corriente de la medicina social privilegia el estudio de la desigualdad social y de la forma en que dicha desigualdad determina los procesos de salud-enfermedad de las poblaciones y de las políticas públicas (López, 1986). Otro trabajo también de amplia influencia en toda Latinoamérica (Bronfman \& Tuirán, 1983; Bronfman et al., 1988) demostró que era empíricamente posible lograr una sofisticada operacionalización del concepto marxista de clase social y utilizarlo tanto para ilustrar como para explicar los diferenciales de mortalidad infantil entre las diversas clases sociales de M éxico. Dicho trabajo se constituyó en un modelo que diversos investigadores aplicaron en sus estudios sobre la desigualdad social y la salud. El concepto como tal, sin embargo, era altamente complejo, lo que resultaba en una cierta desincentivación de los especialistas para utilizarlo. Por ello, pocos años más tarde, Bronfman (Bronfman et al., 1988) propuso una estrategia más simple para clasificar a la población en grupos socio-económicos diferenciados, que ha sido ampliamente retomada en la región.

La corriente de la medicina social se ha ocupado también de problematizar el vínculo salud-trabajo en M éxico (Noriega, 1989). Al hacerlo, diversos autores han señalado la importancia de estudiar al "proceso de trabajo" en sí mismo (Laurell, 1979), y de entenderlo como fuente de "desgaste obrero" (Laurell y M arquez, 1983) con consecuencias directas sobre el proceso salud-enfermedad. Sobre la base de resultados empíricos concretos, los trabajos de Laurell han demostrado que conceptos como "desgaste obrero" y "patrones específicos de desgaste" son más precisos y pertinentes que aquellos como "expropiación absoluta" y "expropiación relativa" de la salud que otros teóricos de la sociología médica marxista habían propuesto en Estados U nidos (N avarro, 1982).

\section{Los sociólogos de la salud pública}

Finalmente, junto a estos abordajes de corte más bien estructural y crítico, una tercera corriente fundacional ha estado más directamente vinculada a la salud pública. Desde mediados de la década de los ochenta, Frenk ha estudiado el comportamiento del mercado de trabajo médico tratando de vincular las características del fenómeno con la clase social de origen de los médicos (Frenk, 1988a; Frenk \& Bashshur, 1983). Para ello, el autor formuló, en primer lugar, una conceptualización quele permitió diferenciar, por una parte, los determinantes generales del mercado de trabajo médico (entre los que incluyó la estructura económica del país, las políticas del Estado, las formas de organización social, las instituciones sociales y la ideología); y por otra parte, los determinantes específicos (tales como la demanda y oferta de servicios médicos, y la educación de los médicos). Posteriormente, el autor realizó una reconstrucción histórica de la evolución de los servicios de salud en este país, así como de la influencia de este factor sobre el mercado de trabajo médico (Frenk et al., 1980). Frenk mostró que la clase social de origen de los médicos se asocia estrechamente tanto al tipo de universidad a la que acceden para estudiar medicina, al tipo de especial ización que eligen realizar, así como al tipo de institución que finalmente los contrata (Frenk, 1984; 1985). Estos trabajos, pioneros en su área, incentivaron el desarrollo de una línea de investigación empírica sobre las características del mercado de trabajo médico en M éxico, que permitieron documentar la existencia de graves contradicciones en el sector médicos subempleados o desempleados, por una parte; y amplios sectores de población sin acceso a servicios de salud, por otra - , así como explorar diversas alternativas de solución a esos desequilibrios (Nigenda et al., 1990; Frenk et al., 1991a).

Al fundar el Instituto Nacional de Salud Pública, Frenk contribuyó a legitimar el desarrollo de trabajos de investigación que, sin ser menos sociológicos que los impulsados por autores como Laurell o $M$ enéndez, permitieron explorar los diversos problemas con abordajes teóricos diferentes a la perspectiva crítica estructural que estos autores propugnan. Las nuevas propuestas de Frenk se dieron en el marco de una serie de importantes debates altamente politizados que sostuvieron diversos representantes de la corriente de la medicina social con él desde la segunda mitad de la década de los ochentas. Cabe citar, a modo de ejemplo, la discusión que se dio en torno a la noción de "transición epidemiológica", introducida en M éxico por los sociólogos de la salud pública a partir del auge del concepto en la literatura internacional (Frenk et 
al., 1989; 1991b). Si bien estas propuestas advertían sobre las peculiaridades de dicha transición en países como M éxico, la crítica, elaborada desde la medicina social, sostenía, entre otras cosas, que el uso de tal concepto era inadecuado en estos contextos toda vez que, en realidad, estos países no transitaban hacia mejores condiciones de salud sino que, en todo caso, veían complicarse su perfil epidemiológico con el repunte de los padecimientos crónicos sin que los padecimientos agudos estuvieran desapareciendo (Laurell et al., 1991). Al final, el balance de este y otros debates fue que era de crucial importancia elaborar marcos teórico-metodológicos que permitieran flexibilizar lo que de otra manera parecía ser un rígido economicismo en la explicación de los problemas y políticas de salud (González-Block \& Frenk, 1986a, 1986b; Fragoso et al., 1986), así como que perspectivas teóricas, aparentemente tan diferentes entre sí, como la salud pública y la medicina social, estaban obligadas a enriquecerse mutuamente de sus aportes y capacidad analítica o afrontar el riesgo de trabajar desde tradiciones académicas interesadas ambas en los problemas de salud de la población y, sin embargo, con muy poco 0 nada que decirse entre sí (Frenk 1988b, 1988c; Eisbenschutz 1988a, 1988b).

\section{La producción contemporánea}

Los debates entre los representantes de la medicina social y los sociólogos de la salud pública continuaron también en la década de los noventa. La firma del Tratado de Libre Comercio entre M éxico, Estados U nidos y Canadá, en 1994, abrió la discusión sobre las posibilidades de incluir a los servicios de salud entre las mercancías a intercambiar entre los tres países. M ientras que los sociólogos de la salud pública veían al Nafta como una oportunidad de modernización, de la que los servicios de salud de M éxico podían beneficiarse (Frenk et al., 1994; Gómez-Dantés et al., 1997), los representantes de la medicina social advirtieron en dicho tratado una clara señal de la tendencia privatizadora del Estado mexicano que consolidaba la desarticulación de las políticas sociales vigentes en décadas pasadas (Laurell \& Ortega 1992).

También en esta década, tras la publicación del informe de 1993 del Banco M undial (1993), la política oficial de salud en M éxico adoptó las propuestas de dicha institución y comenzó a defender la necesidad de "reformar" al sector y de privatizar los servicios, o por lo menos de permitir la participación de capitales privados tanto en la prestación de servicios de salud como en la administración de los fondos de retiro de los trabajadores. Estos cambios dieron lugar a uno de los debates más importantes sobre el tema de salud en el que participan diversos sectores sociales del país. Entre los académicos destacan nuevamente, por una parte, los sociólogos de la salud pública por sus propuestas sobre la manera en que dicha privatización puede hacerse sin, sostienen, menoscabo de los ideales de justicia y equidad (Londoño \& Frenk 1997; Frenk \& González-Block 1992; Lozano 1997), y por otra parte, aquellos que, por el contrario, critican la metodología y el uso de los DALY's (López \& Blanco 1996) y sostienen que la reforma tiende a beneficiar, ante todo, a los detentadores de grandes capitales en perjuicio de los sectores más desprotegidos de la población, dañando así el sentido social de las políticas de salud impulsadas en décadas pasadas (Laurell, 1999; Tetelboin, 1994; López, 1994).

Al mismo tiempo, sin necesariamente tomar parte en esos importantes debates, una segunda generación de trabajos sobre ciencias sociales aplicadas a la salud han hecho su aparición en M éxico a lo largo de la década de los noventas y lo que va de la presente. Algunos de sus autores han sido formados directa o indirectamente en alguno de los tres polos fundacionales mencionados en el apartado anterior. Un buen número de ellos, sin embargo, han sabido ben eficiarse de los aportes de todas esas corrientes, además de que han podido formarse en universidades de Estados U nidos, Canadá, España, Inglaterra o Francia, con especialistas de alto nivel dentro del campo. Ello ha favorecido una suerte de "oxigenación" de la producción académica contemporánea, en tanto que nuevas perspectivas teóricas y nuevos acercamientos metodológicos han enriquecido un escenario antes caracterizado casi exclusi vamente por la perspectiva estructural y los enfoques de la salud pública.

Conviene entonces destinar esta segunda parte del artículo a revisar de manera resumida algunos de los principales aportes de esta nueva generación de trabajos de investigación. Para ello, dividiremos nuestro análisis en tres breves apartados: en el primero, nos referiremos a al gunos de los principales trabajos de carácter teórico o conceptual que han aparecido en los últimos años; en el segundo apartado haremos lo mismo pero centrándonos en los trabajos de 
corte metodológico; y en el tercer apartado nos centraremos en la investigación sustantiva que, como veremos, se refiere a diversos problemas de salud y de utilización de servicios que están concentrando la atención de los científicos sociales especializados en la salud de este país.

\section{A portaciones de orden teórico}

En el nivel de la producción teórica, a partir de los noventas comienzan a aparecer una serie de reflexiones tendientes a revisar el predominio - y los excesos - de los enfoques estructurales en salud (Almada, 1990) así como a explorar las posibilidades de los marcos teóricos que privilegian el análisis de los fenómenos que son mejor observables a un nivel "micro" de análisis. En lo que constituye uno de sus mejores trabajos, M enéndez (1992) señaló que el agotamiento de los grandes sistemas ideológicos se vincula con la incapacidad de los grandes paradigmas teóricos de dar cuenta satisfactoriamente de los diversos niveles de la realidad. Ello, sostiene, ha impulsado una creciente tendencia a buscar en la acción social (prácticas, estrategias, transacciones, etc.) las explicaciones de los fenómenos de salud-enfermedad, más que en las macro-estructuras sociales. El autor advierte, sin embargo, que a menos de que se logre vincular este nivel de análisis con las grandes determinaciones sociales, correremos el riesgo de no trascender el nivel del psicologismo en la explicación de los problemas de nuestro interés.

En el mismo sentido han aparecido investigaciones sobre los conceptos de "calidad de vida" (Blanco et al., 1997), "estilos de vida" y "riesgos" (M artínez, 1993; M enéndez 1998), y “apoyo social" (Castro et al., 1997). Tales trabajos muestran que sólo en la medida en que se preserve el carácter sociológico de tales conceptos - y, por lo tanto, que se preserve el esfuerzo por dilucidar su vinculación con los procesos sociales más generales - se podrá mantener la utilidad de los mismos para el estudio de la determinación de la salud y la enfermedad en la sociedad.

Otros trabajos de carácter teórico que han aparecido en esta década lograron, en su momento, una puesta al día acerca del aporte de la teoría feminista al estudio de los problemas de salud, no sólo en Norte América y Europa, sino también en América Latina (Castro \& Bronfman, 1993; Cardaci, 1998); y, muy destacadamente, significaron una actualización sobre el aporte teórico-metodológico de la perspectiva de género en el estudio de la vinculación entre la condición de la mujer y la salud materno-infantil (Szasz, 1998a).

\section{Aportaciones de orden metodológico}

En el nivel metodológico, dentro del contexto de la sociología médica mexicana, en los últimos años se han publicado varios trabajos con propuestas innovadoras para el estudio social de los fenómenos de la salud y la enfermedad. Desde mediados de la década de los ochentas, dentro de la corriente de la medicina social, se adoptó la perspectiva del "modelo obrero" - originalmente desarrollado en Italia - para realizar investigaciones sobre la salud de los trabajadores. A principios de ésta década se publicó la validación de dicha estrategia en su versión modificada para Latinoamérica. Se trata de una estrategia de investigación que tiene como fundamento la aplicación de entrevistas colectivas, no individuales, entre los trabajadores de la industria, con el triplefin degenerar información sobre los daños a la salud derivados de las condiciones del trabajo, promover la concientización al respecto entre los propios afectados, y formular propuestas colectivas de solución entre los trabajadores (Laurell et al., 1990).

Un segundo desarrollo teórico-metodológico se ha dado en el terreno de la desigualdad social frente a la enfermedad. Bronfman mostró primeramente que las explicaciones convencionales sobre la relación entre variables sociodemográficas (i.e., educación de la madre, nivel socioeconómico, etc.) y mortalidad infantil enfrentan límites muy claros y dejan sin explicar una buena parte del fenómeno (Bronfman, 1992). Posteriormente mostró que las explicaciones más poderosas del problema se logran articulando hábilmente los abordajes cuantitativos y cualitativos. El autor ensayó con éxito una explicación que vincula las determinaciones estructurales de la mortalidad infantil - la posición de clase, ante todo - con los elementos interaccionales de los actores - como la estructura familiar y el funcionamiento de las redes sociales - , y mostró que éstos últimos juegan un papel central tanto en la generación como en la forma de solución de los problemas que desembocan en la mortalidad infantil (Bronfman, 2000).

En la década de los noventas ha tenido lugar un auge de los métodos cualitativos aplicados a la investigación social en salud ( $L$ anger $\& \mathrm{Ni}$ genda, 1995; Szasz \& Lerner, 1996; M ercado \& 
Robles, 1998), al mismo tiempo que han aparecido algunos trabajos de reflexión epistemológica y ética que señalan que la articulación entre los métodos cualitati vos y cuantitativos presenta aún una serie de problemas sin resolver, y que dicha articulación no se logrará adecuadamente a menos que los investigadores mantengan su anclaje en las grandes teorías sociológicas, y abandonen el mero empirismo y los abordajes ateóricos que inundan el campo (Castro, 1996; Castro \& Bronfman, 1999).

\section{Los estudios sustantivos}

El nivel de los estudios sustantivos es el que presenta mayor grado de dificultad frente a cualquier intento de síntesis, no sólo porque es el más numeroso y porque abarca temáticas muy variadas, sino también porque incluye tanto estudios que recurren claramente a la teoría y los métodos de las ciencias sociales, como otros que solamente aluden superficial mente a esas cuestiones. En la práctica, muchos de éstos últimos resultan ser más bien estudios de salud pública débilmente apoyados en las ciencias sociales, pero que difícilmente pueden ser considerados como trabajos de ciencias sociales y salud. En consecuencia, centraremos el resto de nuestro análisis en algunos de los temas que, a nuestro juicio, concentran lo más destacable de la producción sociológica que ha aparecido en los últimos años. Hay que advertir que existen evidentes traslapes entre los diversos campos que aquí mencionaremos y que es sólo por razones de ordenamiento del campo que utilizamos esta clasificación.

\section{- Estudios en el campo de la salud reproductiva}

La salud reproductiva es una cuestión que ha adquirido gran importancia desde la perspectiva de las ciencias sociales aplicadas a la salud en este país (González, 1995; Pérez-Gil et al., 1995; Langer \& Tolbert, 1996). La aparición de diversas compilaciones multi-autorales da cuenta del dinamismo de este campo de conocimiento así como de su diversidad teórico-metodológica (Lartigue\& Avila, 1996; Ojeda, 1999; Stern \& Echárri, 2000; Stern \& Figueroa, 2001). Ello nos imposibilita del todo frente a cualquier pretensión de exhaustividad, por lo que habremos de ser muy selectivos en lo que sigue.

El problema de la mortalidad materna y la maternidad sin riesgos ha sido explorado por diversos autores tratando no sólo de delimitar el alcance del concepto (Reyes, 1994) y de identificar la magnitud del problema (H ernández et al., 1994; Rendón et al., 1993) sino buscando también caracterizar los principales rasgos de orden cultural y simbólico que se le asocian (Elu \& Langer, 1994; Elu, 1993; Castro et al., 2000). El tema sigue siendo objeto de investigación toda vez que la información disponible muestra que se trata de un drama del que desconocemos su magnitud real por efectos del subregistro del que es objeto, y sobre el que no hemos terminado de implementar las políticas más adecuadas para su erradicación.

En el ámbito de la atención del parto se han publicado trabajos que demuestran que aquellas mujeres que reciben al guna forma de apoyo psicosocial durante el embarazo tienen bebés con mejores condiciones de salud (Langer et al., 1993), y aquellas que reciben el apoyo durante la atención del parto tienen una mucho mejor experiencia de este evento que aquellas que son atendidas dentro de las rutinas despersonalizadas de los hospitales públicos (Campero et al., 1998; 2000). También hay estudios que muestran que muchos de los conflictos que se presentan entre prestadores de servicios y usuarias, en el campo de la atención del parto, descansan en la variedad de los prejuicios profesionales y de género que aquellos tienen y que despliegan al realizar su trabajo (Castro, 2000a; Jasis, 2000), o bien en la existencia de diversos paradigmas (religioso, institucional, feminista, etc.) que coexisten en el campo sin necesariamente entenderse mutuamente (Figueroa \& Stern, 2001). Al mismo tiempo, una creciente preocupación por la alta tasa de cesáreas que se practican en México, probablemente muchas de ellas de manera innecesaria, ha dado lugar a diversas investigaciones sobre el tema con miras a identificar mejor los determinantes no médicos, es decir, sociales, de esta práctica médica (Cárdenas, 2000; Campero et al., 2002).

Estrechamente ligado con lo anterior, el tema de los derechos sexuales y reproductivos es objeto de diversas investigaciones en este país. Algunos de los trabajos más relevantes en esta área muestran la distancia que aún existe entre los pronunciamientos internacionales en torno a la salud reproductiva y los derechos de las mujeres y el grado real de asunción y defensa de los mismos - y muy frecuentemente incluso de conocimiento, o de mera información al respecto - que exhiben las mujeres mexicanas (Rivas et al., 1999; Evangelista et al., 2001). Autores como 
Ortíz-Ortega (1999) y Figueroa (1995, 1999), entre otros, han impulsado de manera decisiva el análisis teórico y empírico en torno a la ética y los derechos reproductivos en este país. También ha habido una abundante producción sobre las condiciones sociales que explican las prácticas anticonceptivas y sus determinantes (Lerner \& Quesnel, 1994; Castro et al., 1991); ello, en contraste con las dimensiones de la masculinidad, y el papel de los hombres en los procesos de reproducción, que han comenzado apenas a aparecer (Figueroa, 1998; Rodríguez \& de Keijzer, 1998).

Destaca también el conjunto de trabajos sobre el problema del aborto, muy ligado, naturalmente, a la cuestión de la ciudadanía, los derechos de las mujeres, y el control de los embarazos no deseados. No es aventurado decir quesu principal exponente, M arta Lamas (1993, 2001), ha logrado articular la lucha por la defensa de este derecho proveyéndolo tanto de solidez conceptual como de alternativas concretas para la acción. Investigaciones recientes han documentado la variedad de posiciones ideológicas en torno a este delicado problema en M éxico, rompiendo así con cualquier intento de monopolio oficial de la verdad por parte de las jerarquías eclesiásticas (Ortiz-O rtega, 1994; M ejía \& Careaga, 1996); han recuperado la voz de las propias mujeres, contribuyendo así a combatir la ten dencia a hablar acerca de las mujeres y del aborto, en lugar de escuchar su propia voz (Rivas \& Amuchástegui, 1996); y han documentado el carácter socialmente condicionado de la vivencia del aborto, a partir de las características de las redes sociales, contribuyendo así a documentar las variedades de la experiencia del aborto en contra de cualquier tendencia simplificadora (Castro \& Erviti, 2002).

Las dimensiones sociales de la sexualidad también han sido objeto de investigación en este país, si bien de manera aún incipiente. Destacan los esfuerzos de M inello (1998) por acotar sociológicamente el concepto, así como los de Amuchástegui (2001) y de Rodríguez y de Keijzer (2002). Estos últimos estudios, de corte etnográfico, dan cuenta de los procesos de negociación que tienen lugar entre los jóvenes mexicanos en el ámbito de la sexualidad, así como de los patrones de transformación y cambio que están teniendo lugar en ese espacio. Esos estudios, junto con otros que exploran la sexualidad de los varones (Szasz, 1998b), dan cuenta del carácter profundamente social de la sexualidad humana, y son una fuente de información so- bre un aspecto - la sexualidad entre los diversos grupos de población - que permanecía oscura hasta hace poco (Castro, 1998; Lerner, 1998).

Vinculado cercanamente a este tema, la cuestión de la sexualidad y el embarazo adolescente ha sido objeto de atención de diversos autores en este país (Román et al., 2000; Pedrosa \& Vallejo, 2000; Salcedo, 2000). La investigación social en este campo ha permitido documentar los mitos y dilemas que enfrentan los adolescentes frente al ejercicio de su sexualidad (Rodríguez et al., 1995), al tiempo que se muestra los presupuestos erróneos de los que parten muchos estudios y programas de intervención dirigidos a este grupo de población (Stern \& García, 2001). En otros casos se ha privilegiado el estudio sobre los significados que los adolescentes asocian a su propia sexualidad y al embarazo (Stern, 1996; Tuñón \& Guillén, 1999), y se ha mostrado, incluso, que es mucho más frecuente que los adolescentes se embaracen después de haber abandonado sus estudios, que la inversa - la versión comúnmente creída - esto es, que abandonen sus estudios porque se embarazan ( $M$ enkes \& Suárez, 2002).

En la misma línea, el problema del VIH/SIDA ha sido objeto de estudios sociales de diverso tipo. La mayor parte de los trabajos se refieren al cambio que desarrollan en sus hábitos sexuales los trabajadores mexicanos al migrar a Estados Unidos, que se traduce en un incremento de sus prácticas de riesgo (Bronfman \& M inello, 1995; Salgado, 1998). Sin embargo, una investigación reciente se ha centrado en la migración desde Centroamérica hacia M éxico, proponiendo el concepto de "contextos de vulnerabilidad" para dar cuenta de la situación social que se desarrolla en ciertas estaciones de paso, tales como bares y prostíbulos en ciudades y puertos clave, donde los viajeros pueden incurrir en prácticas de riesgo (Bronfman et al., 2002). O tros trabajos han explorado en detalle la construcción social de la solidaridad y el rechazo familiar y comunitario en torno a las personas con SIDA en M éxico (Castro et al., 1998a, 1998b). Estas últimas son investigaciones de corte cualitativo que exploran con detalle la subjetividad de los individuos con relación a estetema.

Finalmente, la vinculación entre mujeres, trabajo y salud reproductiva ha sido otra área que ha recibido creciente atención en M éxico en los últimos años. Algunos autores han mostrado cómo el acceso diferencial a las oportunidades de trabajo que operan en desventaja de 
las mujeres tienen repercusiones directas en el patrón de morbi-mortalidad de la población infantil (Bronfman \& Gómez-D antés, 1998); en otros casos se ha mostrado que el pretendido efecto negativo que tiene la incorporación de las mujeres al trabajo sobre su salud se debe no al trabajo mismo sino al hecho de que ellas deben vivir diariamente una doble y hasta triple jornada ( como amas de casa, madres y esposas, además de como trabajadoras); por tanto, la eliminación de tales riesgos para la salud pasa por una redistribución de las responsabilidades entre mujeres y hombres (Lara \& Acevedo, 1996; Denman et al., 1995; Garduño \& Rodríguez, 1990). Buena parte de los estudios empíricos sobre este tema se han realizado en las zonas fronterizas, particularmente en el ámbito de las maquiladoras que se ubican a todo lo largo de la frontera entre M éxico y Estados U nidos (Tuñón, 2001). Destacan en este campo las contribuciones de Denman, quien además de haber completado una sistematización sobre los trabajos en esta área (1998), realizó una investigación de campo sobre las prácticas de atención al embarazo de las madres trabajadoras de la industria maquiladora de exportación, así como sobre la influencia de las condiciones de género de dichas mujeres en sus estrategias y recursos de negociación de tales prácticas (2001).

\section{- Estudios sobre violencia contra las mujeres}

Como parte del desarrollo de los temas de los derechos sexuales y reproductivos, la investigación sobre violencia contra las mujeres ha ganado fuerza en los últimos años en Mé$x i c o$. Pero las pioneras en este tema iniciaron sus esfuerzos hace más de diez años en un ambiente mucho menos favorable, en el que era preciso no sólo denunciar el fenómeno del abuso en sí, sino incluso dar los primeros pasos hacia su adecuada conceptualización (Bedregal et al., 1991). Con el correr de la década pasada, el tema comenzó a ser incluido de manera más abierta dentro de la agenda de la salud reproductiva (Saucedo, 1996), hasta que logró establecerse, por pleno derecho, como una prioridad en este campo. Ramírez y U ribe (1993) documentaron tempranamente la magnitud del problema, situándolo en niveles hasta entonces insospechados (prevalencias por arriba de $40 \%$ ). A mediados de la década pasada diversos trabajos argumentaron con solidez la necesidad de considerar a éste como un problema de sa- lud pública (Ríquer et al., 1996; Ramírez \& Patiño, 1996; Díaz-Olavarrieta \& Sotelo, 1997). Otros trabajos se han centrado en al gunas de las formas más específicas de la violencia, por ejemplo la que ocurre en el embarazo (Valdéz \& Sanín, 1996). La investigación en el tema ha permitido desarrollar instrumentos específicos para la medición y comparación del problema con otros países (Castro et al., 2002a). Tales desarrollos han facilitado el estudio del problema de la violencia contra mujeres embarazadas y su comparación con la violencia previa al embarazo (Castro et al., 2002b). Los hallazgos muestran la profunda complejidad del problema y su raigambre social. Por ejemplo, un estudio de percepciones sobre la violencia muestra que en general las mujeres de California (USA) valoran más severamente las diversas formas de violencia contra las mujeres quelas mujeres de M orelos, M éxico (Peek-Asa et al., 2002). Este mismo empuje ha dado paso también a los estudios sobre la masculinidad y la manera en que dicha construcción social se asocia al problema de la violencia. Destacan, en este rubro, los trabajos de Gutmann (2000) y Ramírez (1998). Con todo, un análisis reciente sobre las características de la investigación en este campo ha mostrado que existe una suerte de paradoja: al tiempo que el tema parece agotado ( pues las sucesivas publicaciones sobre violencia contra las mujeres han dejado de aportar datos realmente nuevos), seguimos sin dar con las explicaciones más profundas al problema. Ello se debe, sugieren Castro y Ríquer (2002), a la falta de una orientación sociológica más decidida en este campo, que las investigaciones subsecuentes deberán atender para realmente trascender el nivel de la mera denuncia.

\section{- Estudios sobre subjetividad y salud}

En los últimos años ha cobrado un significativo impulso los estudios cualitativos que buscan elucidar la subjetividad de los individuos en el terreno de la salud, los padecimientos y la enfermedad. En algunos casos, dichos trabajos han propuesto soluciones claramente sociológicas, desde una perspectiva hermenéutica, respecto al problema sobre la manera en que la experiencia subjetiva y los significados en torno a la salud se articulan con la estructura social más general, así como con la desigualdad de género (Castro, 1995; Castro y Eroza, 1998; Castro, 2000). En otros casos, los estudios sobre la experiencia subjetiva se han centrado en los 
padecimientos crónicos, como la diabetes, buscando trasladar el eje de análisis del mero enfoque biomédico 0 , en el otro extremo, del mero enfoque macrosocial y determinista, hacia una perspectiva que incorpora la dimensión personal y subjetiva de los sujetos (M ercado, 1996). Esta perspectiva interpretativa ha enriquecido el estudio de la negociación de la sexualidad y el significado de la virginidad (Arias \& Rodríguez, 1998; Amuchástegui, 1999), así como la vivencia de las mujeres sobre la prevención del cáncer cervicouterino (Castro \& Salazar, 2001). También se ha traducido en investigaciones sugerentes sobre las prácticas médicas de atención a la salud reproductiva en poblaciones indígenas (Gómez \& Forti, 2000), así como sobre la percepción de la maternidad entre diversos grupos de mujeres ( Nuñez, 2000; Nájera et al., 1998). Sin embargo, aún con la riqueza etnográfica de estos trabajos, es posible advertir también aquí las consecuencias de la falta de un abordaje más comprometidamente sociológico: la mayor parte de esas investigaciones suelen quedarse en el nivel del detalle descriptivo, y son muy pocas las que ofrecen una interpretación social o antropológica realmente iluminadora de los testimonios y de las observaciones recolectadas por los investigadores.

\section{- Estudios sobre políticas y utilización de servicios de salud}

Finalmente, un significativo grupo de trabajos sociológicos se ha centrado en el análisis de las políticas de salud, así como en la utilización de los servicios de salud y de medicamentos. Desde un enfoque que critica el rumbo que han tomado las políticas en la actualidad, autores como López y Blanco (1993) y H ernández (1982) han tratado de demostrar cómo la génesis de las políticas actuales se puede rastrear desde la década de los ochentas. Por otra parte, desde una perspectiva que, sin deponer su capacidad crítica, busca identificar los mejores mecanismos para lograr que los resultados de investigación se traduzcan en políticas públicas útiles y eficaces, Bronfman y colaboradores (2000) han impulsado un vigoroso conjunto de reflexiones en el campo de la vinculación entre estos dos espacios de acción.

En el ámbito de la utilización de servicios de salud, se ha documentado la existencia de un claro vínculo entre redes sociales y utilización de servicios (Infante, 1990). También han aparecido ya algunos estudios cualitativos que bus- can conocer la percepción que tienen tanto los usuarios de servicios de salud (Bronfman et al., 1997a) como los propios prestadores de servicios (Bronfman et al., 1997b) acerca del problema de la utilización de los servicios, o bien que buscan identificar el tipo de barreras culturales que dificultan la utilización (Lazcano et al., 1999). Quizás en este renglón la parte menos desarrollada corresponde a la relacionada con la industria farmacéutica y los medicamentos. Recientemente apareció un reporte de una investigación que buscaba explorar los patrones de consumo de medicamentos inseguros en farmacias privadas, sin receta médica (Leyva et al., 1999). A través dela técnica del consumidor ficticio los autores muestran que en este rubro, el de los medicamentos, existe todo un campo que continúa pendiente de ser analizado desde la perspectiva de las ciencias sociales.

\section{Conclusión}

La sociología médica en M éxico es una disciplina que se ha enriquecido con los aportes de muchos científicos sociales que han hecho sus investigaciones en este país en los últimos 25 años. El grueso de la producción aquí reseñada ha salido de instituciones como la U niversidad Autónoma M etropolitana, Ia U niversidad $\mathrm{N}$ acional Autónoma de M éxico, la Universidad de Guadalajara, el Instituto Nacional de Salud Pública, los Centros de Investigación y Estudios Avanzados sobre Antropología Social (Ciesas), El Colegio de M éxico, El Colegio de la Frontera Sur, El Colegio de la Fronteras Norte, El Colegio de Sonora y El Colegio de Michoacán. El papel de este país a nivel latinoamericano se aprecia en la reciente compilación Salud, cambio social y política: perspectivas desde América Latina que, difundida por todo el subcontinente, agotó su primera edición en menos de seis meses (Bronfman \& Castro, 1999). En los años recientes, el predominio casi absoluto que la perspectiva crítica estructural tuvo durante la década de los ochentas ha comenzado a presentar variaciones interesantes. Por una parte, han enriquecido al campo nuevos enfoques interpretativos y nuevas discusiones sobre el alcance y las implicaciones de los diversos abordajes teórico-metodológicos. Por otra parte, han aparecido nuevos enfoques críticos - como los estudios de género y salud o los trabajos sobre las determinaciones sociales de la experiencia de los padecimientos, por mencionar sólo algunos ejemplos - quehan 
retomado el espíritu crítico del enfoque estructural y, en muchos sentidos, lo han enriquecido. El aporte que el conjunto de estos trabajos ha hecho a la comprensión de los problemas de salud ha alcanzado ya un punto crítico: hoy en día, gran parte de la investigación en salud pública se apoya en los principales conceptos y métodos desarrollados por las ciencias sociales. Citemos a modo de ejemplo la tendencia a utilizar técnicas como la encuesta o los grupos focales para recabar información; o la tendencia a considerar las desigualdades socioeconómicas, - las características del núcleo familiar y social para explicar las variaciones que presentan los fenómenos que se analizan.

No es aventurado afirmar que en más de un sentido la sociología médica mexicana se encuentra en la frontera del conocimiento a nivel mundial. Varios de sus trabajos - por ejemplo, la operacionalización exitosa del concepto marxista de clase social en la década pasada y su aplicación en el estudio de diversos problemas de salud; o la incorporación de variables cualitativas, como la estructura familiar y el funcionamiento de las redes sociales, para el estudio de la desigualdad social frente a diversos proble- mas de salud - constituyen verdaderas novedades sobre las que no se reporta equivalente alguno en la literatura internacional.

Sin embargo, en los últimos años han disminuido los debates entre diferentes escuelas que caracterizaron a la sociología médica mexicana en los años ochenta. Ello puede deberse, en parte, al relativo desdibujamiento de las corrientes fundacionales, habida cuenta de que la nueva generación de investigadores en este campo ha sabido beneficiarse de los principales conceptos y métodos de cada una de ellas, deponiendo cualquier forma de ortodoxia o parroquialismo. Pero también puede ser aviso de un posible abandono del espíritu de discusión e intercambio que debe caracterizar a toda empresa académica, abandono que ha tenido lugar, quizás, en aras de la comodidad. Si este fuera el caso, una de las tareas centrales de esta disciplina es la reactivación de este recurso, junto con la necesidad de focalizar la investigación en los problemas más urgentes. A menos de que se recupere la vigilancia entre pares a la que debemos someter toda producción académica, estaremos condenando nuestros esfuerzos a una medianía que nuestra disciplina y nuestra realidad social no merecen.

\section{Referencias bibliográficas}

Aguirre-Beltrán G 1963. M edicina y magia. El proceso de aculturación en la estructura colonial. Instituto $\mathrm{Na}$ cional Indigenista, M éxico, 443pp.

Aguirre-Berltrán G 1986. Antropología médica. Ciesas, M éxico.

Almada I 1986. Las ciencias sociales en salud en M éxico, pp. 133-142. In E N unes (ed.). Ciencias sociales y salud en América Latina. Organización Panamericana de la Salud, M ontevideo.

Almada I 1990. Siete tesis equivocadas sobre salud y sociedad. Cuadernos M édico-Sociales 52:15-25.

Amuchástegui A 1999. Dialogue and the negotiation of meaning: constructions of virginity in M exico. Culture, Health and Sexuality 1(1):79-93.

Amuchástegui A 2001. Virginidad einiciación sexual en M éxico. Experiencias y significados. Edamex, The Population Council, M éxico, 436pp. 
Arias R \& Rodríguez M 1998. "A puro valor mexicano". Connotaciones del uso del condón en hombres de clase media de la Ciudad de M éxico, pp. 319-340. In Lerner S. (ed.). Varones, sexualidad y reproducción. El Colegio de M éxico, M éxico

Banco M undial 1993. Informe sobre el desarrollo mundial. Invertir en Salud. Banco M undial, Washington.

Bedregal X, Saucedo I \& Ríquer F 1991. Hilos, nudos y colores en la lucha contra la violencia hacia las mujeres. Ediciones Cicam, M éxico.

Blanco J, López O, Rivera JA \& Rueda F 1997. Una aproximación a la calidad de vida. Límites conceptuales y metodológicos. Estudios de Antropología y Biología 8 : 433-448.

Boltvinik J, Santos-Burgoa C, Almada-Bay I, M endoza R $\&$ Chávez AM 1983. N ecesidades esenciales en M éxico. Salud. Coplamar, Siglo XXI, M éxico, 452pp.

Bronfman M 1992. Infant mortality and crisis in M exico. International Journal of $\mathrm{H}$ ealth Services 22(1):157167.

Bronfman M 2000. Como se vive se muere. Familia, redes sociales y mortalidad infantil. CRIM/UNAM, Cuernavaca.

Bronfman M, Castro V, Guiscafré H, Castro R \& Gutiérrez $\mathrm{G}$ 1998. La medición de la desigualdad: una estrategia metodológica. Análisis de las características socioeconómicas de la muestra. Archivos de Investigación M édica 19(4):351-360.

Bronfman M, Castro R, Zúñiga E, M iranda C \& Oviedo J 1997a. Del 'cuánto' al 'por qué': la utilización de los servicios de salud desde la perspectiva de los usuarios. Salud Pública de M éxico 39(5):442-450.

Bronfman M, Castro R, Zúñiga E, M iranda C \& Oviedo J 1997b. 'H acemos lo que podemos': Ios prestadores de servicios frente al problema de la utilización. SaIud Pública de M éxico 39(6):546-553.

Bronfman M \& Castro R (eds.) 1999. Salud, cambio social y política: perspectivas desde América Latina. Edamex, M éxico.

Bronfman M \& Gómez-Dantés H 1998. La condición de la mujer y la salud infantil, pp. 89-125. In JG Figueroa (ed.). La condición de la mujer en el espacio de la salud. El Colegio de M éxico, M éxico

Bronfman M, Langer A \& Trostle J (eds.) 2000. De la investigación en salud a la política: la difícil traducción. INSP, M anual M oderno, M éxico, 178pp.

Bronfman M , Leyva R \& N egroni M 2002. M obile population and HIV/AIDS in Central America and M exiCo: research for action. AIDS (en prensa).

Bronfman M , Lombardi C \& Victora CG 1988. Operacionalização do conceito de classe social em estudos epidemiológicos. Revista de Saúde Pública 22(4):253265.

Bronfman M \& Minello N 1995. Hábitos sexuales de los migrantes temporales mexicanos a los Estados U nidos de América. Prácticas de riesgo para la infección por VIH, pp. 3-89. In M Bronfman et al., SIDA en Mé xico. M igración, adolescencia y género. Información Profesional Especializada, M éxico.

Bronfman M \& Tuirán R 1983. La desigualdad social ante la muerte: clases sociales y mortalidad en la niñez. Cuadernos M édico Sociales 29:53-75.

Campero L et al. 1998. 'Alonel wouldn't have known what to do': a qualitative study on social support during labor and delivery in M exico. Social Science and M edicine 47(3):395-403.
Campero $L$ et al. 2000. Apoyo psicosocial durante el parto: experiencias y percepciones de las madres, pp. 263-300. In Stern C. \& Echárri C. (comps.). Salud re productiva y sociedad. Resultados de investigación. El Colegio de M éxico, M éxico.

Campero $L$ et al. 2002. N on-clinical factors affecting cesarean section in women who received birth education: a study in M exico. American Journal of Public $\mathrm{H}$ ealth, forthcoming.

Campos R (ed.) 1992. La antropología médica en M éxico. Instituto M ora y Universidad Autónoma M etropolitana, M éxico, (2 vols.).

Cardaci D 1998. Nuevos entramados: familia, salud y organizaciones de mujeres, pp. 243-265. In JG Figueroa (ed.). La condición de la mujer en el espacio de la salud. El Colegio de M éxico, M éxico.

Cárdenas R 2000. La práctica de la cesárea en las áreas urbanas de M éxico, pp. 301-328. In C Stern \& C Echárri (comps.). Salud reproductiva y sociedad. Resultados de investigación. El Colegio de M éxico, M éxico.

Castro M C \& Salazar G 2001. Elementos socioculturales en la prevención del cáncer cérvicouterino. Un estudio en Hermosillo, Sonora. El Colegio de Sonora, Hermosillo, 230pp.

Castro R 1995. The subjective experience of health and illness in Ocuituco: a case study. Social Science and M edicine 41(7):1.005-1.021

Castro R 1996. En busca del significado: supuestos, alcances y limitaciones del análisis cualitativo, pp. 57-85. In I Szasz \& S Lerner (coords.). Para comprender la subjetividad. Investigación cualitativa en salud reproductiva y sexualidad. El Colegio de M éxico, M éxico.

Castro R 1998. 'Uno de hombre con la mujer es como una corriente eléctrica': subjetividad y sexualidad entre los hombres de Morelos. Debate Feminista 9(18): 105-130.

Castro R 2000a. Formas de precariedad y autoritarismo presentes en la vivencia de la reproducción en el área rural de M orelos, pp. 33-66. In C Stern \& C Echárri (comps.). Salud reproductiva y sociedad. Resultados de investigación. El Colegio de M éxico, M éxico.

Castro R 2000. La vida en la adversidad. El significado de la salud y la enfermedad en la pobreza. CRIM, Cuernavaca.

Castro R, Bronfman M \& Loya M 1991. Embarazo y parto entre la tradición y la modernidad: el caso de O cuituco. Estudios Sociológicos 9(27):583-606.

Castro R \& Bronfman M 1993. Teoría feminista y sociología médica: bases para una discusión. Cadernos de Saúde Pública 9(3):375-394

Castro R \& Bronfman M 1999. Problemas no resueltos en la integración de métodos cualitativos y cuantitativos en la investigación social en salud, pp. 49-64. In M Bronfman \& R Castro (coords.). Salud, cambio social y política. Perspectivas desde América Latina. Edamex, M éxico.

Castro R \& Riquer F 2002. La investigación sobre violencia contra las mujeres en América Latina: entre el empirismo ciego y la teoría sin datos. Cadernos de Saúde Pública (en prensa).

Castro R, Garcia L, Ruiz A \& Peek-Asa C 2002a. Developing an index to measure violence against women for comparative studies between M exico and the U nited States. Journal of Family Violence (en prensa).

Castro R, Peek-Asa C \& Ruiz A 2002b. Violence against women before and during pregnancy in M exico: The 
role of lifetime violence exposure. American Journal of Public $\mathrm{H}$ ealth (en prensa).

Castro R, Campero L \& Hernández B 1997. La investigación sobre apoyo social en salud: situación actual y nuevos desafíos. Revista de Saúde Pública 31(4): 425-35.

Castro R \& Eroza E 1998. Research notes on social order and subjectivity: individuals' experience of susto and fallen fontanelle in Central M exico. Culture, M edicine and Psychiatry 22(2):203-230.

Castro R \& Erviti J 2002. Las redes sociales en la experiencia del aborto. El caso de las mujeres de Cuernavaca (M éxico). Cadernos de Saúde Pública (en prensa).

Castro R et al., 1998a. AIDS-related illness trajectories in M exico: results from a qualitative study in two marginalized communities. AIDS Care: Psychological \& Socio-medical aspects of AIDS/HIV 10(4):583-598.

Castro R, Eroza E, Orozco E, H ernández JJ \& Aggleton P. 1998b. Family responses to HIV/AIDS in M exico. Social Science and M edicine 47(10):1.473-1.484.

Castro R, Campero L, H ernández L \& Langer A. 2000. A study on maternal mortality in M exico through a qualitative approach. Journal of Women's H ealh \& Gender-Based M edicine 9(6):679-690.

Denman C 1998. Salud y maquila: acotaciones del campo de investigación en vista de las contribuciones recientes. Relaciones 74(19):75-100.

Denman C 2001. Prácticas de atención al embarazo de madres trabajadoras de una maquiladora en $\mathrm{N}$ ogales, Sonora, M éxico. Tesis de doctorado, El Colegio de M ichoacán, M éxico.

Denman C, Balcázar H \& Lara F 1995. Factors associated with work-related accidents and sickness of maquiladora workers: the case of N ogales, Sonora, M exico. International Journal of $\mathrm{H}$ ealth Services 25(3): 489-502.

Diaz-Olavarrieta C \& Sotelo J 1997. Domestic violence in M exico. JAM A 275(24):1.937-1.941.

Eibenschutz C1988a. ¿Quétan moderna es la salud pública moderna?. N exos 123:74-75.

Eibenschutz C1988b. ¿Dónde está la polémica?. N exos 126:78-79.

Elu M C 1993. La luz enterrada: estudio antropológico sobre la mortalidad materna en Tlaxcala. FCE, M éxico, 150pp.

Elu, M C \& Langer A (eds.) 1994. M aternidad sin riesgos en M éxico. IM ES y Comité Promotor de la Iniciativa por una M aternidad sin Riesgos en M éxico, M éxico, 244pp.

Evangelista A, Tuñón E, Rojas M \& Limón F 2001. Derechos sexuales y reproductivos entre mujeres jóvenes de una comunidad rural de Chiapas. Revista M exicana de Sociología 63(2):139-165.

Figueroa JG 1995. A proximación al estudio de los derechos reproductivos. Reflexiones: sexualidad, salud y reproducción 8. El Colegio de M éxico, M éxico.

Figueroa JG 1998. Algunos elementos para interpretar la presencia de los varones en los procesos de salud reproductiva. Cadernos de Saúde Pública 15(1):87-96.

Figueroa JG 1999. Derechos reproductivos y el espacio de las instituciones de salud: algunos apuntes sobre la experiencia mexicana, pp. 147-190. In A Ortiz-Ortega (comp.). Derechos reproductivos de las mujeres: un debate sobre justicia social en M éxico, Edamex y UAM $X$, M éxico.
Figueroa JG \& Stern C (coords.) 2001. Encuentros y desencuentros en la salud reproductiva. Políticas públicas, marcos normativos y actores sociales. El Colegio de M éxico, M éxico, 236pp.

Fragoso A, Velázquez S\& Hermida J 1986. El contrahorizonte de la salud. N exos 106:46-47.

Frenk J 1984. La estratificación social en la educación médica mexicana. Salud Pública de M éxico 26(3): 484-491.

Frenk J 1985. Efectos del origen social y de la socialización profesional sobre las preferencias vocacionales de los internos de medicina en M éxico. Educación M édica y Salud 19(4):426-451.

Frenk J 1988a. Social origin, professional socialization and labor market dynamics: the determinants of career preferences among medical interns in M exico. (Serie Perspectivas en Salud Pública). Instituto Nacional de Salud Pública, M éxico.

Frenk J 1988b. La modernización de la salud pública. Nexos 122:57-58.

Frenk J 1988c. De la autonomía al autismo. N exos 124:7576.

Frenk J \& Bashshur R 1983. Career preferences and perceptions of the medical labor market among M exican interns. Social Science and M edicine 17(11):693-704.

Frenk J, Bobadilla JL, Sepúlveda J \& López-Cervantes M . 1989. Health transition in middle-income countries: new challenges for health care. Health Policy and Planning 4(1):29-39.

Frenk J et al., 1991a. Patterns of medical employment: a survey of imbalances in urban Mexico. American Journal of Public Health 81(1):23-29.

Frenk J, Bobadilla JL, Stern C, Frejka T, \& Lozano R 1991b. Elements for a theory of the health transition. Health Transition Review 1:21-38.

Frenk J, Hernández H \& Alvarez L 1980. El mercado de trabajo médico. Gaceta M édica de M éxico 116(4): 187-195.

Frenk J \& González-Block M A 1992. Primary care and reform of health systems: a framework for the analysis of Latin American experiences. H ealth Services M anagement Research 5(1):32-43.

Frenk J et al. 1994. Consequences of the N orth American free trade agreement for health services: a perspective from M exico. American Journal of Public $\mathrm{H}$ ealth 84(10):1.591-1.597.

García de Alba J, M unck V, Salcedo AL, Vargas LA, \& Garro T 1998. Consensus analysis: high blood pressure in a M exican Barrio, pp. 197-210. In VC M unck \& EJ Sobo (eds.). U sing methods in the filed. A practical introduction and casebook, Altamira Press, W alnut Creek.

Garduño M A \& Rodríguez JC 1990. Salud y doble jornada: taquilleras del metro. Salud-Problema 20:41-45.

Gómez T \& Forti S 2000. Prácticas médicas de atención a la salud reproductiva en una comunidad otomí del estado de Querétaro, pp. 67-96. In Stern C. \& Echárri C. (comps.). Salud reproductiva y sociedad. Resultados de investigación. El Colegio de M éxico, M éxico.

Gómez-Dantéz O, Frenk J \& Cruz C 1997. Commerce in health services in N orth America within the context of the N orth American Free Trade Agreement. Revista Panamericana de Salud Pública 1(6):460-465.

González S (ed.) 1995. Las mujeres y la salud. El Colegio de M éxico, M éxico.

González-Block M A \& Frenk J 1986a. El horizonte de la salud. N exos 100:59-61. 
González-Block M A \& Frenk J 1986b. El contrahorizonte del economicismo. N exos 106:41-42.

Gutmann M C 2000. Ser hombre de verdad en la Ciudad de M éxico. Ni macho ni mandilón. El Colegio de M éxico, M éxico, 394pp.

Hernández B, Chirinos J, Romero M \& Langer A 1994. Estimating maternal mortality in rural areas of $M$ exico: the application of an indirect demographic method. International Journal of Gynecology \& O bstetrics 46: 285-289.

Hernández H 1982. Historia de la participación del estado en las instituciones de atención médica en M éxico 1935-1980, pp. 49-96. In F Ortíz Quezada (ed.). Vida y muerte del mexicano, vol. 2. Folios Ediciones, M éxico.

Infante C 1990. Utilización de servicios de atención prenatal: influencia de la morbilidad percibida y de las redes sociales de ayuda. Salud Pública de M éxico 32(4):419-429.

Jasis M . 2000. A palabras de usuaria... ¿oídos de doctor? Una exploración sobre las necesidades de salud reproductiva de las mujeres en La Paz, Baja California, pp. 97-146. In C Stern \& C Echárri (comps.). Salud reproductiva y sociedad. Resultados de investigación, El Colegio de M éxico, M exico.

Lamas M 1993. La bioética: proceso social y cambio de valores. Sociológica 8(22):135-147.

Lamas M . 2001. M ovimiento feminista y discurso político: los derechos sexuales y reproductivos en la construcción de una ciudadanía moderna, pp. 177-194. In JG Figueroa \& C Stern (coords.). Encuentros y desencuentros en la salud reproductiva. Políticas públicas, marcos normativos y actores sociales. El Colegio de M éxico, M éxico.

Langer A, Garcia C, Leis T, Reynoso S \& Hernández B 1993. El apoyo psicosocial durante el embarazo como estrategia para promover la salud del recién nacido. Revista de Investigación Cliníca 45:317-28.

Langer A \& Nigenda G (eds.) 1995. M étodos cualitativos para la investigación en salud pública. (Serie Perspectivas en Salud Pública 20). Instituto Nacional de Salud Pública, Cuernavaca.

Langer A \& Tolbert K (eds.) 1996. M ujer, sexualidad y salud reproductiva en M éxico. The Population CouncilEdamex, M éxico.

Lara M A \& Acevedo M C 1996. Incorporación de la mujer al trabajo remunerado: repercusiones para su salud reproductiva, pp. 119-151. In A Langer \& K Tolbert (eds.). M ujer: sexualidad y salud reproductiva en M éxico. The Population Council y Edamex, M éxico.

Lartigue T \& Avila H (comps.) 1996. Sexualidad y reproducción humana en M éxico. ( 2 vols.). Universidad Iberoamericana y Plaza y Valdes Editores, M éxico.

Laurell AC 1974. Sociología médica: una bibliografía comentada. Revista M exicana de Ciencia Política 75: 105-111.

Laurell AC 1975. M edicina y capitalismo en M éxico. Cuadernos Políticos 5:80-93.

Laurell AC 1979. Work and health in M exico. International Journal of H ealth Services 9(4):543-568.

Laurell AC 1982. La salud-enfermedad como proceso social. Revista Latinoamericana de Salud 2:7-25.

Laurell AC 1989. Social analysis of collective health in Latin America. Social Science and M edicine 28(11): 1.183-1.191.

Laurell AC 1999. The M exican social security counter- reform: pensions for profit. International Journal of H ealth Services 29(2):371-391.

Laurell AC \& M árquez M 1983. El desgaste obrero en M é xico. Proceso de producción y salud. Editorial Era, Mé xico.

Laurell AC, Noriega M , López O \& Ríos V 1990. La experiencia obrera como fuente de conocimiento. Confrontación de resultados de la encuesta colectiva e individual. Cuadernos M édico Sociales 51:5-26.

Laurell AC \& Ortega M 1992. The free trade agreement and the M exican health sector. International Journal of $\mathrm{H}$ ealth Services 22(2):331-337.

Laurell AC, Franco S, Nunes E \& Breilh J 1991. Debates en medicina social. O pas-Alames, Quito.

Lazcano-Ponce $E$ et al. 1999. Barriers to early detection of cervical-uterine cancer in M exico. Journal of Women's Health 8(3):399-408.

León-Portilla M 1999. Bernardino de Sahagún. Pionero de la antropología. UNAM - El Colegio Nacional, M éxico, 261pp.

Lerner S (ed.) 1998. Varones, sexualidad y reproducción. El Colegio de M éxico, M éxico, 436pp.

Lerner S \& Q uesnel A 1994. Las transacciones institucionales y la pluralidad de trayectorias reproductivas. Estudios Demográficos y de Desarrollo U rbano 9(3): 543-578.

Leyva R, Erviti J, Bronfman M \& Gasman N 1999. Consumo de medicamentos en farmacias privadas: los medicamentos inseguros, pp. 493-508. In M Bronfman \& R Castro (coords.). Salud, cambio social y política: perspectivas desde América Latina. Edamex México.

Londoño JL \& Frenk J 1997. Structured pluralism: towards an innovative model for health system reform in Latin America. Health Policy 41(1):1-36.

López D 1986. La salud desigual en M éxico, Siglo XXI Editores, M éxico.

López 0 1994. La selectividad en la política de salud, pp. 33-60. In AC Laurell (ed.). N uevas tendencias y alternativas en el sector salud. Universidad Autónoma M e tropolitana-Xochimilco, M éxico.

López O \& Blanco J 1996. Las necesidades de salud y las propuestas de solución. Notas para la discusión, pp. 79-97. In C García \& H Hernández (eds.). M ortalidad, salud y discurso demográfico. CRIM (UNAM), Cuernavaca.

López 0 \& Blanco J 1993. La modernización neoliberal en salud. M éxico en los ochenta. UAM -X, M éxico, 145pp.

López-Austin A 1971. Textos de medicina Náhuatl. SEP/ Setentas, M éxico, 230pp.

López-Austin A 1980. Cuerpo humano eideología. Las concepciones de los antiguos nahuas. UNAM , M éxico, (2 vols.).

Lozano R 1997. El peso de la enfermedad en M éxico: avances y desafíos, pp. 23-61. In J Frenk (ed.). O bservatorio de la salud: necesidades, servicios, políticas. Fundación M exicana para la Salud, M éxico.

Lozoya X \& Zolla C (eds.) 1983. La medicina invisible. Introducción al estudio de la medicina tradicional en M éxico. Folios Ediciones, M éxico, 303pp.

M artínez C 1993. Sobrevivir en Malinalco. La salud al margen de la medicina. El Colegio de M éxico, Universidad Autónoma M etropolitana-Xochimilco, Mé xico.

M ejía M C \& Careaga G 1996. Concepciones alternativas sobre sexualidad, reproducción, anticoncepción y 
aborto, pp. 349-406. In G Careaga, JG Figueroa \& C M ejía (comps.). Ética y salud reproductiva. Ed. M iguel Angel Porrúa, M éxico.

M ellado V, Zolla C \& Castañeda X 1989. La atención al embarazo y el parto en el medio rural mexicano. CIESS, M éxico, 162pp.

M enéndez E 1978. El modelo médico y la salud de los trabajadores, pp. 11-53. In F Basaglia. La salud de los trabajadores. Ed. Nueva Imagen, M éxico.

M enéndez E 1983. H acia una práctica médica alternativa. H egemonía y autoatención (gestión) en salud. Cuadernos de la Casa Chata 86.

$M$ enéndez E 1984. Estructura y relaciones de clase y la función de los modelos médicos. Nueva Antropología 6(23):71-102.

M enéndez $E$ 1985a. El modelo médico dominante y las limitaciones y posibilidades de los modelos antropológicos. Desarrollo Económico 24(96):593-604.

M enéndez E 1985b. Saber "médico" y saber "popular": el modelo médico hegemónico y su función ideológica en el proceso de alcoholización. Estudios Sociológicos 3(8):263-296.

M enéndez E 1990. Antropología médica. Orientaciones, desigualdades y transacciones. Cuadernos de la Casa Chata 179. Ciesas, M éxico.

M enéndez E 1992. Grupo doméstico y proceso salud/enfermedad/atención. Del 'teoricismo' al movimiento continuo. Cuadernos M édico Sociales 59:3-18.

$M$ enéndez E 1998. Estilos de vida, riesgos y construcción social. Conceptos similares y significados diferentes. Estudios Sociológicos 16(46):37-67.

M enkes C \& Suárez L 2002. Embarazo y sexualidad adolescente. In F Lozano (coord). VI reunión demográfica. vol.1, Somede-Crim-Unam (en prensa).

M ercado FJ 1996. Entre el infierno y la gloria. La experiencia de la enfermedad crónica en un barrio urbano. Universidad de Guadalajara, M éxico, 354pp.

M ercado FJ \& Robles L 1998. Investigación cualitativa en salud. Perspectivas desde el occidente de M éxico. Universidad de Guadalajara, M éxico, 313pp.

M inello N 1998. De las sexualidades. Un intento de mirada sociológica, pp. 35-48. In I Szasz \& S Lerner (comps.). Sexualidades en M éxico. Algunas aproximaciones desde la perspectiva de las ciencias sociales. El Colegio de M éxico, M éxico.

M ódena ME 1990. M adres, médicos y curanderos: diferencia cultural eidentidad ideológica, Ediciones de la Casa Chata Núm. 37, M éxico, 229pp.

Nájera AG et al. 1998. M aternidad, sexualidad y comportamiento reproductivo: apuntes sobre la identidad de las mujeres, pp. 275-305. In JG Figueroa (comp.). La condición de la mujer en el espacio de la salud, El Colegio de M éxico, M éxico.

Navarro V 1976. M edicine under capitalism. Prodist, N ew York.

Navarro V 1982. The labor process and health: a historical materialist interpretation. International Journal of H ealth Services 12(1):5-29.

Nigenda G, Frenk J, Robledo-Vera C, Vázquez LA \& Ramírez C 1990. Los sistemas locales de salud y el mercado de trabajo médico: resultados de un estudio de preferencias de ubicación geográfica. Educación M édica y Salud 24(2):115-135.

Noriega M 1989. Problemas teórico-metodológicos de la investigación sobre salud en el trabajo. Salud-Problema 17:9-13.
Nuñez AE 2000. La percepción de la maternidad en un grupo de mujeres rurales, pp. 235-262. In Stern C \& Echárri C (comps.). Salud reproductiva y sociedad. Resultados de investigación. Colmex, M éxico.

Ojeda N (coord.) 1999. Género, familia y conceptualización de la salud reproductiva en M éxico. El Colegio de la Frontera Norte, M éxico, 453pp.

Ortiz-Ortega A (ed.) 1994. Razones y pasiones en torno al aborto. Una contribución al debate. The Population Council y Edamex, M éxico, 334pp.

Ortíz-Ortega A (comp.) 1999. Derechos reproductivos de las mujeres: un debate sobre justicia social en M éxico. Edamex, UAM-X, M éxico, 375pp.

Pedrosa LA \& Vallejo M 2000. Entorno social, comportamiento sexual y reproductivo en la primera relación sexual de adolescentes estudiantes de escuelas públicas y privadas, pp. 177-198. In Stern C \& Echárri C (comps.). Salud reproductiva y sociedad. Resultados deinvestigación. El Colegio de M éxico, M éxico.

Peek-Asa C, Garcia L, M cArthur D \& Castro R 2002. Severity of intimate partner abuse indicators as perceived by women in M exico and the U nited States. Women \& Health 35(2/3):165-180.

Pérez-Gil SE, Ramírez JC \& Ravelo P (eds.) 1995. Género y salud femenina. Experiencias de investigación en M éxico, Ciesas-UG-INN, M éxico, 328pp.

Ramírez JC 1998. Violencia masculina: algo más que gobernarse a sí mismo. La Ventana 7:225-251.

Ramírez JC \& U ribe G 1993. M ujer y violencia: un hecho cotidiano. Salud Pública de M éxico 35(2):148-160.

Ramírez JC \& Patiño M C 1996. M ujeres de Guadalajara y violencia doméstica: resultados de un estudio piloto. Cadernos de Saúde Pública 12(3):405-409.

Reyes S 1994. M ortalidad materna en M éxico. Instituto M exicano del Seguro Social, M éxico.

Ríquer F, Saucedo I \& Bedolla P 1996. Agresión y violencia contra el género femenino: un asunto de salud pública, pp. 247-287. In A Langer \& K Tolbert (eds.). $M$ ujer: sexualidad y salud reproductiva en M éxico. The Population Council-Edamex, M éxico.

Rivas M \& Amuchástegui A 1996. Voces e historias sobre el aborto. The Population Council-Edamex, M éxico, 125pp.

Rivas M, Amuchástegui A \& Ortiz-Ortega A 1999. La negociación de los derechos reproductivos en M éxico, pp. 257-370. In A Ortíz-Ortega (comp). Derechos reproductivos de las mujeres: un debate sobre justicia social en M éxico. Edamex-UAM-X, M éxico.

Rodríguez $G$, Amuchástegui $A$, Rivas $M \&$ Bronfman $M$ 1995. M itos y dilemas de los jóvenes en tiempos del SIDA, pp. 91-200. In M Bronfman et al. SIDA en México. M igración, adolescencia y género. Inf. Profesional Especializada, M éxico.

Rodríguez $\mathrm{G} \&$ de Keijzer B 1998. La noche se hizo para los hombres: las regulaciones sexuales del cortejo en una comunidad cañera. Debate Feminista 9(18):237-266.

Rodríguez G \& de Keijzer B 2002. La noche se hizo para los hombres. Sexualidad en los procesos de cortejo entre jóvenes campesinas y campesinos. The Population Council-Edamex, M éxico, 250pp.

Romám R, Carrasco ME, Abril E \& Cubillas MJ 2000. N oviazgo y embarazo: una mirada a las trayectorias de amor y conflicto en mujeres adolescentes embarazadas, p. 147-176. In Stern C \& Echárri C (comps.). Salud reproductiva y sociedad. Resultados de investigación. El Colegio de M éxico, M éxico. 
Sahagún FB 1989. Historia general de las cosas de la N ueva España. Conaculta, M éxico.

Salcedo AL 2000. La experiencia del embarazo y su atención en adolescentes de estratos medio, popular y marginal, pp.199-234. In Stern C \& Echárri C (comps.). Salud reproductiva y sociedad. Resultados de investigación. El Colegio de M éxico, M éxico.

Salgado N 1998. M igración, sexualidad y Sida en mujeres de origen rural: sus implicaciones psicosociales, pp. 155-174. In I Szasz \& S Lerner (comps.). Sexualidades en M éxico. Algunas aproximaciones desde la perspectiva de las ciencias sociales. El Colegio de M éxico, M éxico.

Saucedo I 1996. Violencia doméstica y salud: conceptualización y datos que existen en M éxico. Revista Perinatología y Reproducción Humana 10(2):100-110.

Simoni JJ, Vargas LA \& Casillas LE 1983. M erolicos y educación de salud. Boletín de la O ficina Sanitaria Panamericana 94(5):461-472.

Stern C 1996. El embarazo en adolescentes como problema público: una visión crítica. Salud Pública de México 39(2):137-143.

Stern C \& Echárri C (comps.) 2000. Salud reproductiva y sociedad. Resultados de investigación. El Colegio de M éxico, M éxico, 403pp.

Stern C \& Figueroa JF (coords.) 2001. Sexualidad y salud reproductiva. Avances y retos para la investigación. El Colegio de México, M éxico, 420pp.

Stern C \& García E 2001. Hacia un nuevo enfoque en el campo del embarazo adolescente, pp. 331-364. In Stern C \& Figueroa JF (coords.). Sexualidad y salud reproductiva. A vances y retos para la investigación. EI Colegio de M éxico, M éxico.

Szasz I 1998a. Alternativas teóricas y metodológicas para el estudio de la condición de la mujer y la salud materno-infantil, pp. 313-331. In JG Figueroa (comp.). La condición de la mujer en el espacio de la salud. El Colegio de M éxico, M éxico.

Szasz I 1998b. Los hombres y la sexualidad: aportes de la perspectiva feminista y primeros acercamientos a su estudio en M éxico, pp. 137-162. In Lerner S. (ed.). Varones, sexualidad y reproducción. El Colegio de México, M éxico
Szasz I \& Lerner S (eds.) 1996. Para comprender la subje tividad. Investigación cualitativa en salud reproductiva y sexualidad. El Colegio de M éxico, M éxico, 256pp.

Tetel boin C 1994. El problema de los recursos humanos, pp. 89-110. In AC Laurell (ed.). Nuevas tendencias y alternativas en el sector salud. Universidad Autónoma M etropolitana-Xochimilco, M éxico.

Tuñón E (coord.) 2001. M ujeres en las fronteras: trabajo, salud y migración (Belice, Guatemala, Estados U nidos y M éxico). El Colegio de la Frontera N orte, Ecosur, El Colegio de Sonora, Plaza y Valdés Editores, M éxico, 333pp.

Tuñón E \& Guillén C 1999. Embarazo adolescente en dos generaciones de madres Tabasqueñas: una exploración a sus vivencias, en Esperanza Tuñón (ed.). Gé nero y salud en el sureste de M éxico. UADY-Ecosur, San Cristóbal de las Casas (en prensa).

Valdéz R \& Sanín LH 1996. La violencia doméstica durante el embarazo y su relación con el peso al nacer. Salud Pública M ex 38:352-362.

Vargas LA 1973. El embarazo y el parto en el M éxico prehispánico. Anales de Antropología X:297-310.

Vargas LA 1978. Acceptance and rejection of medical practices of different cultures, pp. 14-16. In B Velimirovic (ed.). M odern medicine and medical anthropology in the US-M exico border population. Pan-American H ealth Organization, Scientific Publication 359, Washington, D.C.

Vargas LA 1989. M edical anthropology in M éxico. Social Science and M edicine 28(2):1.343-1.349.

Vargas LA 1991. Una mirada antropológica a la enfermedad y el padecer. Gaceta M édica de M éxico 127(1): 3-5.

Vargas LA 1993. El conocimiento médico en el M éxico prehispánico, pp. 11-29. In H. Aréchiga y J. Somolinos (comps). Contribuciones mexicanas al conocimiento médico. Fondo de Cultura Económica, M éxico.

Vargas LA, Kemper RV \& Casillas LE 1993. Réseaux migratoires et santé, pp. 62-71. In Y. Preiswerk \& J. Vallet (eds.). Vers un ailleurs prometteur... L'émigration, une réponse universelle á une situation de crise? Presses Universitaires de France, Cahiers de l'I.U.E.D., Paris.

Zolla C, del BosqueS, Tascón A \& M ellado C. 1988. M edicina tradicional y enfermedad. CIESS, M éxico.

Artigo apresentado em 10/9/2002

A provado em 10/10/2002

Versão final apresentada em 20/10/2002 